\title{
Inconsistency of performance on neglect subtype tests following acute right hemisphere stroke
}

\author{
R.H. HAMILTON, ${ }^{1}$ H.B. COSLETT,,${ }^{1,2}$ L.J. BUXBAUM, ${ }^{2}$ J. WHYTE, ${ }^{2}$ AND M.K. FERRARO ${ }^{2}$ \\ ${ }^{1}$ Department of Neurology, University of Pennsylvania, Philadelphia, Pennsylvania \\ ${ }^{2}$ Moss Rehabilitation Research Institute, Thomas Jefferson University, Philadelphia, Pennsylvania
}

(Received December 11, 2006; Final Revision June 26, 2007; Accepted June 27, 2007)

\begin{abstract}
Hemispatial neglect has been conceptualized as having dissociable and potentially clinically relevant subtypes. However, the question of whether patient performance on neglect subtype measures is consistent over time remains largely unanswered. We examined changes in performance over time on measures of motor, perceptual, and personal neglect in 21 patients with neglect from acute right hemisphere stroke. Patients were assessed on three occasions, separated by at least one week, using a lateralized target test, lateralized response test, and modified fluff test. Across three testing timepoints, $18(85.7 \%)$ patients changed subtype performance patterns at least once. In $13(61.9 \%)$ of these patients, inconsistency between timepoints was not adequately accounted for by recovery. On initial testing, seven, patients $(33.3 \%)$ demonstrated more than one neglect subtype symptom; by the third testing timepoint none of the patients demonstrated multiple symptoms. In the setting of acute stroke, performance on three measures of neglect symptoms is inconsistent across time. However, the distribution of neglect subtype symptoms appears to become more discrete over time. These findings complicate our understanding of the pathophysiology and potential prognostic value of neglect subtypes, and suggest that treatment decisions based on subtype performance assessed at a single timepoint, may be of limited utility. (JINS, 2008, 14, 23-32.)
\end{abstract}

Keywords: Attention, Hemispatial neglect, Hemisensory neglect, Variability, Brain infarction, Perceptual disorders

\section{INTRODUCTION}

Hemispatial neglect, defined as failure to report, respond, or orient to meaningful or novel stimuli on the contralesional side of space (Heilman et al., 1985), is a common and often profoundly disabling consequence of unilateral brain damage, particularly of the right hemisphere. Left hemispatial neglect is frequently associated with lesions to the right temporal-parieto-occipital junction, but is it also observed after damage to other regions in the parietal cortex, frontal lobes, thalamus, and basal ganglia (Karnath et al., 2001; Vallar \& Perani, 1986). The disorder is present in a large proportion of right hemisphere stroke patients, and has been associated with poor clinical outcome as indicated by more limited mobility and longer hospitalizations (Paolucci et al., 2001) as well as higher degrees of longterm functional disability and family burden (Buxbaum et al., 2004; Katz et al., 1999).

Correspondence and reprint requests to: H. Branch Coslett, M.D., Department of Neurology, Hospital of the University of Pennsylvania, 3400 Spruce Street, Philadelphia, PA 19104. E-mail: hbc@mail.med.upenn.edu
Neglect has been conceptualized as having discrete subtypes characterized by constellations of symptoms based on categorical distinctions (Heilman et al., 2000). Commonly recognized distinctions include (1) the perceptual versus intentional quality of neglect symptoms; (2) the sensory modality of neglect symptoms; and (3) the region of space affected by neglect. Patients with perceptual neglect show a selective unawareness of contralesional stimuli, whereas patients with intentional neglect exhibit directional hypokinesia for actions into and toward contralesional hemispace (Coslett et al., 1990; Heilman et al., 2000) despite preserved awareness. Neglect can differentially impact various sensory modalities, including vision, audition, and touch (Sterzi et al., 1993). It may also affect regions of contralesional space differently; in some patients deficits may be restricted to contralesional body parts (personal neglect), whereas in others it may encompass contralesional space beyond the body. Neglect extending beyond the body may affect specific parts of contralesional space, including areas within reaching distance (peripersonal neglect) or regions beyond reaching distance (extrapersonal neglect) (Bisiach 
et al., 1986; Guariglia \& Antonucci, 1992; Vallar, 2001). Neglect can also impair mental representation of contralesional extrapersonal objects and environments (representational neglect) (Bisiach \& Luzzatti, 1978)

The consistency of patient performance within putative neglect subtype categories is important for both theoretical and clinical reasons. Observations of similarities and differences between neglect subtypes and associated brain injuries may shed light on their underlying cognitive processes and neural substrates. Some data also suggest that the prognosis of patients with neglect may depend on the subtype of neglect that they exhibit (Appelros et al., 2004). Previous work and its potential implications have been based on the assumption that neglect subtypes correspond to stable categories that are reflected in patient performance. However the important question of whether patient performance within neglect subtypes remains consistent over time has not yet been thoroughly investigated. We therefore evaluated a subset of patients with acute right hemisphere strokes previously identified in a larger study by Buxbaum et al. (2004) on three separate occasions using tests designed to identify three subtypes-intentional, attentional, and personal neglect.

\section{METHODS}

\section{Subjects}

Twenty-one patients participated in the study. They were drawn from a larger cohort of 86 acute right hemisphere stroke patients (Buxbaum et al., 2004). Patients were considered eligible for the study if they had sustained a hemorrhagic or ischemic right hemisphere stroke within three months of testing. Subjects all demonstrated clinical signs of neglect on a battery of behavioral measures (see later), and were tested on at least three separate occasions. Individuals with prior head injuries, left hemispheric strokes, other neurologic disorders, or DSM-IV Axis I disorders were excluded. Inclusion was also based on 3 behavioral criteria: (1) language comprehension adequate to understand the instructions for each test, (2) visual and auditory acuity and motor function sufficient to perform each test, and (3) attention and behavioral control adequate for a 90-minute testing session.

The mean age of the 21 subjects was 69.1 years (Range $=$ $38-85$ years old; $S D=11.3$ years). Subjects were righthanded and had an average of 7.3 years of education ( Range $=1-16$ years; $S D=4.7$ years). There were 6 males and 15 females. On neurologic examination one subject had a contralesional visual field cut, 10 had visual extinction, whereas 8 demonstrated tactile extinction. All subjects showed some degree of left-sided weakness, with 18 demonstrating no measurable grip strength in the left hand using a dynamometer. Clinical imaging used to aid in the initial diagnosis of stroke revealed a heterogeneous group of lesions leading to neglect. One infarct affected the right frontal lobe only, another affected the frontal lobe and underlying subcortical structures, and two damaged both frontal and temporal lobes. Two infarcts affected the right parietal lobe only, three involved the right frontal and parietal cortex, and two affected the parietal cortex and underlying subcortical structures. Five strokes affected subcortical structures (basal ganglia, thalamus, and internal capsule) predominantly. All four lobes of the right hemisphere were affected in three patients, whereas one infarct involved the frontal, temporal, and parietal cortex and subcortical structures. One stroke involved only occipital and temporal cortex. The average period between the onset of stroke and initial testing in these patients was 21.6 days (Range $=5-37$ days; $S D=8.6$ days). On average the second testing session occurred 29.6 days after stroke (Range 13-44 days; SD 8.5 days), and the third testing point occurred 67.4 days after stroke (Range $=27-287$ days; SD 60.7 days). Testing sessions were at least 1 week apart and the average interval between sessions was 17.1 days $(S D=25.79$ days).

\section{Clinical Neglect Battery}

Five tests comprised the initial clinical neglect battery for neglect. These were the Bells Test and four subtests of the Behavioral Inattention Test (BIT, Wilson et al., 1987): Letter Cancellation, Picture Scanning, Menu Reading, and Line Bisection.

\section{Bells Test}

Patients were required to cancel 35 bells presented in a dense array with 282 other stimuli on $8.5 \times 11^{\prime \prime}$ paper. Left-right accuracy difference scores were calculated.

\section{Letter cancellation}

Patients were required to cancel the letters E and R (20 each) presented among 150 other letters on $8.5 \times 11^{\prime \prime}$ paper. The differences between the numbers of targets canceled on the left and right were calculated.

\section{Picture scanning}

Patients were required to name objects in an $11^{\prime \prime} \times 14^{\prime \prime}$ photograph of items used in grooming and self-care. There are two versions of the subtest; one was assigned to each patient for the duration of testing. We calculated the percent of targets named on the left and right of the total possible (three on the left in both versions; four on the right in version A, and five on the right in version B), and then calculated the difference between the left and right accuracy percentage scores.

\section{Menu reading}

Patients were required to read 24 words presented in four columns. Left-right difference scores in accuracy were calculated. 


\section{Line bisection}

Patients were required to indicate the center of each of three lines, each 20.5-cm long. The mean distance of responses (in $\mathrm{mm}$ ) from the true midpoint was calculated for each patient.

\section{Determination of neglect}

The presence of neglect was defined by performance below cut-off scores on at least one of the five clinical neglect tests. For the Bells Test, Letter cancellation, and Menu reading, patients were considered to exhibit neglect if their leftright difference scores were greater than $20 \%$ of the total number of items on each side of the array. Patients were considered to exhibit neglect on the Picture Scanning Task if the difference between the percent of items named on each side of the array was greater than 20 percentage points. The cut-off score for line bisection was determined by scoring guidelines provided for the Behavioral Inattention Test (Wilson et al., 1987). Using this definition neglect was present in nearly $50 \%$ of the right hemisphere stroke patients studied previously by Buxbaum et al. (2004), which was consistent with previous reports of neglect prevalence (Stone et al., 1992).

\section{Study Measures}

Patients were seated at a desk in a quiet, dedicated examination room for all testing. Subjects were seated approximately $57 \mathrm{~cm}$ from a computer monitor used for testing. Examiners sat beside patients to the right and administered all tests in a standardized manner. Simple directions were read aloud to the patients from a standard script. Order of test administration was randomized across subjects; for each subject the order of tests was consistent across sessions.

\section{Lateralized Response Test}

This test was designed to assess intentional neglect. Subjects viewed three horizontally-arrayed $5 \mathrm{~mm}$ dots (subtending $\sim 0.5^{\circ}$ of visual angle) each separated by $4 \mathrm{~cm}\left(\sim 4^{\circ}\right.$ of visual angle) on a computer monitor, and were asked to fixate on the central dot. A tester visually monitored subjects' fixation on the central target; trials in which the tester noted deviation of gaze from the central target were eliminated. Participants began each trial with their right forefinger on the table top at midline with respect to the keyboard and their body. After $2000 \mathrm{~ms}$, a left-, right-, or downwardpointing arrow replaced the central dot (Fig. 1a), indicating how subjects should respond. In each trial the arrow persisted until a subject responded. Subjects responded by pressing keys that were either on the left (keys Q, W, E, A, S, and D), central (keys I, O, P, K, L, and ;), or right (keys 7, $8,9,4,5$, and 6) on a partially-shielded keyboard (Buxbaum \& Permaul, 2001). Subject response immediately initiated the next trial.
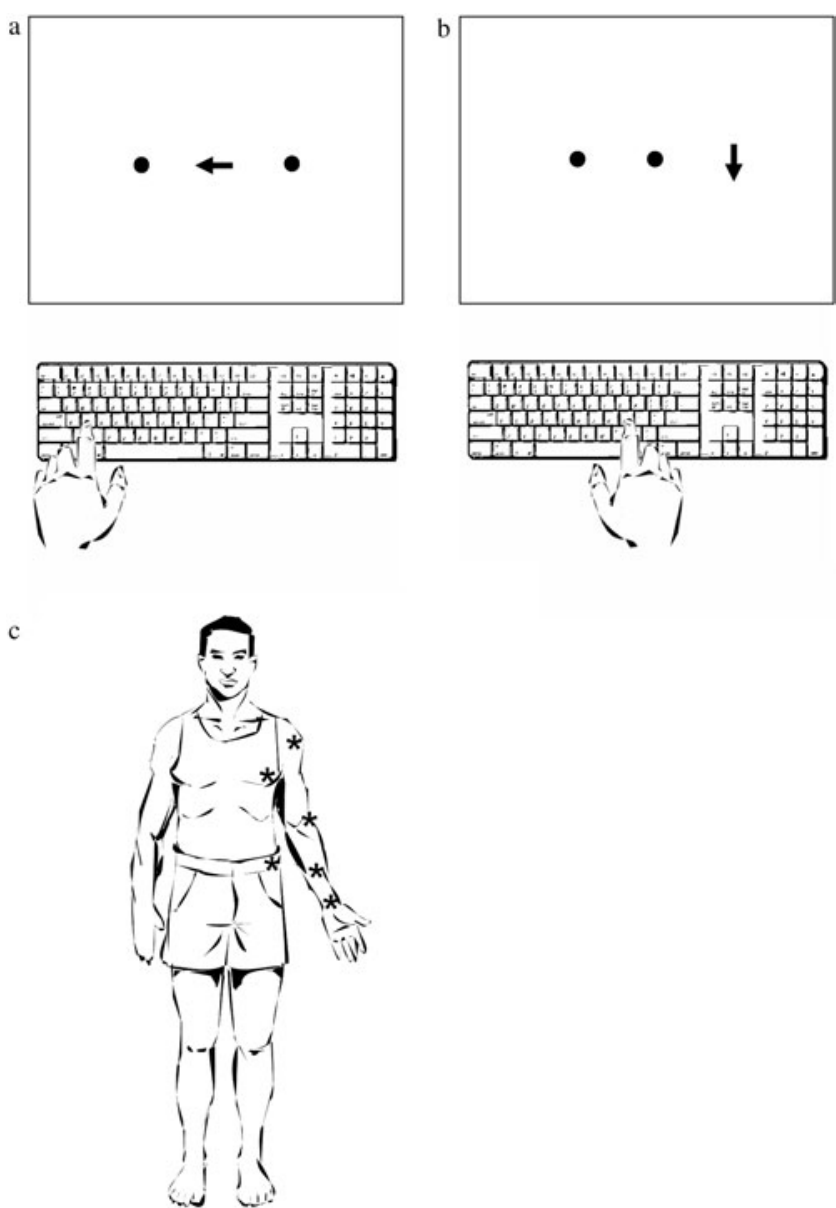

Fig. 1. a, Lateralized Response Test. After fixating on a central point, subjects responded by pressing a key on the left, right, or center of the keyboard as indicated by a central arrow. b, Lateralized Target Test. After fixating on a central point, subjects responded to arrows appearing either on the right or left side of the screen by pressing a key at the center of the keyboard. c. Modified Fluff Test. Subjects attempted to locate and remove cotton balls that had been placed on their left shoulder, chest, elbow, forearm, wrist, and hip. (*'s indicate approximate location of cotton balls.)

\section{Lateralized Target Test}

This test was designed to assess attentional neglect. Participants viewed an array of dots identical to those in the lateralized response test $(5 \mathrm{~mm}$ in width, separated by $4 \mathrm{~cm})$, and were asked to fixate on the center dot. After $2000 \mathrm{~ms}$, a downward-pointing arrow replaced one of the three dots (Fig. 1b), and persisted until the subject responded. Participants responded by pressing the spacebar on the computer keyboard when a left- or right-sided arrow was detected (Buxbaum \& Permaul, 2001). Subject response initiated the next trial.

In the lateralized response and lateralized target tests, nine practice trials were followed by 20 randomized test trials. After eliminating responses $<100 \mathrm{~ms}$ or $>10,000 \mathrm{~ms}$, a mean left-right difference score was calculated for each 
participant based on reaction times. Less than $2 \%$ of responses for each patient were discarded on this basis.

\section{Modified Fluff Test}

Personal neglect was assessed by adhering six cotton balls on a blindfolded participant's left side at the shoulder, chest, elbow, forearm, wrist, and hip (Fig. 1c). On removal of the blindfold, the participant was instructed to locate and remove the cotton balls. The number of detected targets was tallied (0-6). (Cocchini et al., 2001 for description of a similar test). Patients' ability to detect and remove cotton ball was only tested for targets placed on the left side and removed with the right hand because most patients had severe leftsided weakness.

\section{Definitions of Abnormal Performance}

Because normal subjects exhibit no asymmetry on the Lateralized Target, and Lateralized Response Tests and perform at ceiling on the Modified Fluff Test, performance on the three tests was compared to the mean performance of the 86 acute right hemisphere stroke subjects who had performed the tests at least once (Buxbaum et al., 2004). The demographics of this larger population were similar to that of our sample cohort (Mean age $=67.4$ years old, range $37-89$ years; mean education $=10.7$ years, range $1-20$ years; days post onset of stroke $=19.4$ days, range $=5-41$ days). As in the previously reported study, the 20th percentile of performance of this larger group was used as the cutoff criterion for classifying the performance of our cohort of patients as normal or abnormal on each of the three tests.

\section{Classification of Neglect Subtypes}

Subjects were designated as having "intentional" neglect if they performed abnormally on the Lateralized Motor Response Test, "attentional" neglect if they performed abnormally on the Lateralized Stimulus Test, and "personal" neglect if they performed abnormally on the Fluff Test. Possible combinations of neglect subtypes included: attentional+intentional, attentional+personal, intentional+personal, and attentional+intentional+personal neglect.

All data was obtained in compliance with the regulations of the ethics review committees of the participating institutions.

\section{RESULTS}

\section{Performance Patterns Over Time}

We considered a patient to have a stable neglect subtype if he or she demonstrated a consistent pattern of performance on our neglect measures over the three testing periods. Using this criterion, only 3 of 21 subjects (14.3\%) demonstrated
Table 1. Neglect subtype performance for all subjects

\begin{tabular}{rccccc}
\hline \hline Patient & Time 1 & Time 2 & Time 3 & Stable? & Recovery? \\
\hline 1 & All & Int/Per & Int & \\
2 & All & None & Att & & \\
3 & Att/Per & Int/Per & Per & & \\
4 & Att/Per & None & Att & \\
5 & Att/Per & None & None & \\
6 & Att/Int & Int & None & \\
7 & Att/Int & Int & None & \\
8 & Att & Att & Att & \\
9 & Att & Int & Per & \\
10 & Per & None & Int & \\
11 & Int & Int & None & \\
12 & None & Att & Att & \\
13 & None & Att/Int & None & \\
14 & None & Per & None & \\
15 & None & Int & Int & \\
16 & None & Int & Int & \\
17 & None & Int & None & \\
18 & None & None & Int & \\
19 & None & None & Int & \\
20 & None & None & None & $レ$ \\
21 & None & None & None & $レ$ & \\
\hline \hline
\end{tabular}

Rows represent individual patients. "Timel" = testing timepoint 1; "Time2" = testing timepoint 2; "Time3" = testing timepoint 3; "Att" = attentional neglect; "Int" = intentional neglect; "Per" = personal neglect; "All" = all three subtypes; "None" = no subtypes; "Stable" = stable pattern of performance across timepoints; "Recovery?" = $\boldsymbol{レ}$ indicates pattern of performance potentially consistent with recovery.

stable performance. One patient demonstrated a consistent pattern of attentional neglect, whereas two other patients performed normally on all three measures during each session. The remaining 18 patients $(85.7 \%)$ changed neglect subtype at least once over the course of the three testing sessions. The neglect subtype classification for all 21 patients for each of the three testing sessions is listed in Table 1.

One potential explanation for the apparent volatility of neglect subtypes is that patients' neglect improved over time. To address this issue, improvement was operationally defined as a decrease in the number of neglect tests on which subjects performed abnormally over the course of the three test administrations; for example, the performance of a subject who performed abnormally on the attentional+intentional tests on administration one, only the attentional test on administration 2 and no tests on administration 3 was considered to be consistent with improvement. Using this criterion, the performance of 5 of the 18 patients (38.5\%) was consistent with improvement (Table 1), whereas 13 subjects $(61.5 \%)$ changed neglect subtypes in ways that were not explained by improvement. For example, 11 of 21 subjects (52.4\%) exhibited neglect on at least one subtest in the third session but had performed normally on all three tasks during the first or second session. To further investigate the possibility of improvement, mean subject performance on the three subtype tests was compared across test timepoints. Three repeated measures ANOVAs were performed 
with performance on each test the dependent measure and time (time 1 , time 2 , or time 3 ) repeated within subjects. No significant effect was observed for either the Lateralized Target $(F=2.52, p=.107)$ or the Fluff Tests $(F=1.869$, $p=.182$ ), indicating no significant change in the performance on either of these tests. There was a significant main effect of time for the Lateralized Response Condition ( $F=$ 4.062; $p=.034$ ). Post hoc testing revealed a trend toward improved mean performance between the first and second timepoints $(t=-1.89, p=.073)$ and a trend toward declining performance between the second and third timepoints ( $t=1.80, p=.089$ ). There was, however, no difference between mean subject performance at the first and third timepoints $(t=-.126, p=.901)$. Thus, using an inclusive definition of improvement, the changes of subtype for most subjects were not readily attributable to recovery.

In addition to improvement, a second potential explanation for variability is that the performance of some subjects may have straddled the cutoff values used to operationally define abnormal performance, such that minor changes in performance might have resulted in changes in categorization from normal to abnormal performance, or vice versa. We therefore sought to exclude patients whose performance across testing timepoints on the lateralized target and response tests spanned the 20th percentile but remained within a five-point window the cutoff values (15th to 25 th percentiles). Because the Fluff Test was measured on a sixpoint ordinal scale, we looked for patients whose performance on this test remained within a one-point window of the cutoff. We identified no patients whose variability could be attributed to closely straddling the cutoff values.

A third potential contributor to the inconsistency of patients' performance is variability in either the interval between stroke onset and initial testing or between the three testing timepoints. We therefore compared the mean duration of these intervals in patients who demonstrated consistent or potentially improved performance (combined $n=8$ ) to those of patients who demonstrated inconsistent performance $(n=13)$ across the testing timepoints. Student $t$-tests comparing the intervals between stroke onset and timepoints one $(t=1.24, p=.229)$, two $(t=1.19 ; p=.247)$, and three $(t=.154 ; p=.874)$. A comparison of the interval between the first and the third timepoint was also nonsignificant $(t=1.41 ; p=.175)$.

\section{Multiple versus Single Neglect Subtypes}

The number of subjects in each subtype category at each of the three testing timepoints is shown in Table 2. On initial testing of 21 patients, four $(19.0 \%)$ were considered to have a single neglect subtype (attentional, intentional, or personal), whereas seven of the 21 patients (33.3\%) demonstrated more than one neglect symptom. By the third testing timepoint, patients either demonstrated deficits on one $(57.1 \%)$ or zero $(42.9 \%)$ subtype measures; none showed combined neglect subtypes. We further quantified this change by dividing patients into two groups: those who had showed
Table 2. Distribution of neglect subtype symptoms across three testing timepoints

\begin{tabular}{lccc}
\hline \hline Subtype & Time 1 & Time 2 & Time 3 \\
\hline Attentional & 2 & 2 & 4 \\
Intentional & 1 & 7 & 6 \\
Personal & 1 & 1 & 2 \\
Attent/Intent & 2 & 1 & 0 \\
Attent/Personal & 3 & 0 & 0 \\
Intent/Personal & 0 & 2 & 0 \\
All abnormal & 2 & 0 & 0 \\
None abnormal & 10 & 8 & 9 \\
$(N=21)$ & & &
\end{tabular}

"Time1" = testing timepoint 1 ; "Time2" = testing timepoint 2 ; "Time3"= testing timepoint 3; "Attentional" = attentional neglect; "Intentional" = intentional neglect; "Personal" = Personal neglect; "Attent/Intent" = attentional neglect + intentional neglect; "Attent/Personal" = attentional neglect + Personal neglect; "Intent/Personal" = intentional + Personal; "All" = all three subtype symptoms; "None abnormal" = no subtype symptoms.

abnormalities on either one or none of the neglect subtype tests (isolated subtypes group) and those who performed abnormally on multiple neglect subtype tests (combined subtypes group). Comparison of these two groups between timepoint 1 and timepoint 3 using Fisher Exact Test demonstrated a significant shift $(P=.001)$ from the combined subtypes group to the isolated subtypes group. Thus, there emerged a shift over time from a spectrum of varied and overlapping neglect symptoms to a profile of discrete seemingly non-overlapping deficits.

On initial testing 5 out of 21 patients $(23.8 \%)$ were considered to have intentional neglect, either in isolation or in combination with other subtypes. Nine patients $(42.9 \%)$ had attentional neglect and six $(28.6 \%)$ had personal neglect. At the second timepoint there was an increase in patients with intentional neglect (10 patients; $47.6 \%$ ) but a decrease in patients with attentional neglect (3 patients; $14.3 \%$ ) or personal neglect (3 patients; 14.3\%). This difference in trend between intentional neglect and other neglect subtypes was significant $\left(\chi^{2}=25.2 ; p<.001\right)$. Similarly, between testing timepoints one and three the number of patients with intentional neglect increased from five $(23.8 \%)$ to six $(28.6 \%)$, whereas the number of patients considered to have attentional neglect decreased from $9(42.9 \%)$ to $4(19.1 \%)$ and the number of patients with personal neglect decreased from $6(28.6 \%)$ to $2(9.5 \%)$. This change was also significant $\left(\chi^{2}=10.3 ; p<.01\right)$. Thus a disproportionate number of patients showing lateralized deficits in intention emerged over time.

\section{DISCUSSION}

We evaluated a cohort of acute right hemisphere stroke patients with neglect on three occasions using tests designed to assess three aspects of hemispatial neglect. There were two main findings. The first was that neglect subtype per- 
formance was highly inconsistent. Several lines of evidence demonstrated that this instability could not be explained by spontaneous recovery or practice effects. The second finding was that the distribution of neglect symptoms changed markedly over time. Whereas a number of patients initially demonstrated multiple neglect subtype symptoms, all of them eventually settled into one of the putative neglect subtypes postulated by other investigators.

\section{Inconsistency of Subtype Performance}

We conceive of two potential explanations for the marked variability of patient performance in our cohort: either neglect subtype symptoms are inconsistent following acute stroke, or the tests used to assess these symptoms are unreliable. In line with the notion that neglect symptoms are variable, our findings support earlier investigations that have demonstrated the vulnerability of neglect patients to variable performance on spatial tests. Small and Ellis (1994) administered the Behavioral Inattention Test (BIT) on a weekly basis to 10 patients with left-sided neglect following stroke. They noted periods of normal performance interleaved with periods of neglect in patients who had not recovered by the end of the study, suggesting marked variability of performance over time. Bailey and colleagues (2004) further investigated the test-retest variability for unilateral neglect patients who were tested three times in an hour using the cancellation task, line bisection, and the baking tray task. They found that the test-retest stability of these measures was relatively low for patients with mild or moderate neglect. In fact, variability in the performance of patients with neglect has been proposed by some to be a more robust marker of neglect than a lateralized spatial bias. Anderson and colleagues (2000) used a response time task to demonstrate that highly variable performance between trials was more closely associated with neglect than a consistent rightward bias. Pizzamiglio and colleagues (2000) found that patients with a lateralized spatial bias on a line bisection task showed increased variability bidirectionally on a body-centered task.

Not all evidence, however, supports the instability of neglect patients' performance. Levy and colleagues (1995) found little fluctuation in the performance on the Visual Neglect Recovery Index (VNRI) in 22 patients with acute stroke and 19 with chronic stroke who were tested twice in one day. Furthermore, Barrett and colleagues (1999) reported a case in which a patient demonstrated stability of motorintentional neglect through an eight-week treatment period, as well as a deficit-specific, albeit paradoxical, response to treatment with bromocriptine. However, while consistent deficit-specific performance may characterize some patients' symptoms, our results suggest that most patients experience significant variability in their performance on neglect measures over time.

A number of factors may contribute to variability of performance in acute right hemisphere stroke patients. Nonlateralized deficits in attention, which are often prominent in patients with unilateral neglect, can influence the severity of lateralized symptoms (Coslett et al., 1987; Duncan et al., 1999; Hjaltason et al., 1996; Robertson, 2001) Levels of arousal and alertness have been shown to have significant effects on lateralized spatial attention in normal adults (Fimm et al., 2006). Right hemisphere frontal and parietal structures may play a specialized role in the maintenance of tonic alertness (Sturm \& Willmes, 2001), and the interaction between overall arousal and lateralized spatial attention is underscored by the finding that noradrenergic agents and other CNS stimulants can improve lateralized deficits in patients with right hemisphere lesions and neglect (Malhotra et al., 2006). Deficits in the ability to maintain tonic alertness may have thus contributed to our subjects' variable performance.

The testing environment itself may have contributed to inconsistent performance. Factors such as the location of the tester in relation to the subject can significantly influence performance (Coslett, 1999). Whereas an attempt was made to control the testing environment, variations in unsuspected and therefore uncontrolled environmental cues or testing procedures may have influenced subjects' responses.

Patients may have also shown variability caused by problems reorienting attention within or between tests. Prefrontal and parietal cortices have been implicated in test switching (Brass et al., 2005). Patients with right hemisphere lesions, particularly lesions of the parietal cortex, have difficulty disengaging attention from targets in order to fixate attention on new ones (Losier \& Klein, 2001; Posner et al., 1984), and patients with neglect are known to perseverate on targets (Na et al., 1999; Rusconi et al., 2002). Deficits of attentional disengagement and test switching may have interfered with subject performance not only within trials but also across trials or even between tests at a given testing timepoint.

Aside from the possible instability of neglect subtypes, the other potential explanation for inconsistent performance over time is that our measures may have been insensitive or unreliable. Prior investigations have reported differing sensitivities for similar neglect measures applied to similar patient populations, suggesting that these measures may capture symptoms unreliably. For example, in a systematic review of studies, Bowen and colleagues (1999) found that the frequency of neglect in right hemisphere stroke patients ranged from $13 \%$ to $82 \%$. The test-retest reliability of our subtype tests is unknown, and our finding that many subjects had new symptoms emerging weeks to months after their strokes may suggest unreliability of the tests, because spontaneous development of new subtype symptoms is an unusual finding. However, our finding that subtype performance in our cohort becomes more discrete over time suggests that the tests are capturing relevant patterns of patient performance rather than just noise from poor test-retest reliability.

Methodological limitations of the study may have contributed to unreliability in our measures. Cutoff scores were used to distinguish normal from abnormal performance; 
selection of higher or lower thresholds would have affected the number of subjects considered to have a specific subtype of neglect at each timepoint. However, no subjects demonstrated performance that straddled these cutoffs, suggesting that the threshold scores appropriately targeted the most abnormal performers. Variability between subjects in testing intervals is another methodological limitation that does not explain the inconsistency in subject performance, because there was no difference in these intervals between patients who demonstrated stable or improved performance and those who did not. A third methodological issue to consider is the comparability of our tests. Because RTs are the dependent measures for the lateralized attention and lateralized response tasks but number of errors on a six-point scale is the dependent measure for the modified Fluff Test, the tests may not have been equally sensitive to the presence of neglect subtypes or to changes in symptom severity over time. However, whereas these scaling issues may limit our ability to make comparisons between subtypes they do not account for the emergence of previously absent symptoms in the majority of subjects.

Another reason that our measures may not have been reliable is that they may not be valid measures of neglect subtypes. One limitation of many clinical evaluations of neglect, including this study, is that the choice of measures is based on face validity, which may not reflect high sensitivity or specificity for the symptoms that they are designed to test. In addition, it may be the case that variability of neglect subtype performance reflects poor construct validity in the conceptualization of subtypes themselves. It has been shown that performance on one neglect subtype task is poorly correlated with performance on tasks designed to evaluate the same subtype deficit (Harvey et al., 2002). It could be the case that attention, intention, and other concepts currently used to make the categorical distinctions that define subtypes only crudely approximate the true distinctions that characterize different presentations of neglect.

Importantly, regardless of whether neglect subtypes performance is inconsistent because subtype symptoms are unstable following acute brain injury or because the tests themselves are unreliable, our results prompt reconsideration of whether early categorization of neglect into subtype syndromes-which is often operationally defined by single measures of performance on tests similar to ourshas clear diagnostic and prognostic value. This observation has important implications for stroke rehabilitation and recovery from neglect. At least one recent study has suggested that patients with neglect of peripersonal space experience complete resolution of this symptom less often than those who demonstrate either neglect of far space or of personal space (Appelros et al., 2004). Patients in this study were initially tested between two and four weeks following stroke, which approximates the period over which patients in our study were initially tested. The results of our study, however, suggest that the prognostic value of acute neglect patients' subtype symptoms may be complicated by the instability of their early performance. It might be the case, for instance, that subtype diagnoses are more predictive when made later in the course of stroke, or that very large data sets are needed to overcome early noisy subtype performance.

\section{Emergence of Discrete Subtypes}

It is intriguing that, whereas the neglect symptoms experienced by individual patients in our study varied dramatically over time, the spectrum of neglect symptoms experienced by our cohort progressed from an overall pattern of combined deficits to one of discrete subtype symptoms. In light of the specialized role of the right hemisphere in both general arousal and lateralized spatial attention, one plausible explanation is that the patterns of performance seen in acute right hemisphere stroke patients result from a dynamic interplay between nonlateralized arousal deficits and deficits of lateralized spatial attention. Nonlateralizing arousal deficits may have contributed considerably to noisy, highly variable patient performance, whereas the segregation of patients into discrete subtype performance profiles over time suggests the influence of dissociable lateralized deficits.

Another plausible explanation for the emergence of discrete performance patterns is that neglect subtypes may not be an immediate consequence of brain injury, but rather an emergent property of neural systems that are adapting to operate optimally in the presence of a brain lesion. A similar argument has been advanced to explain the evolution of symptoms in aphasia (Welbourne \& Lambon Ralph, 2005a, Welbourne \& Lambon Ralph, 2005b). A third possible explanation is that there may have been a reduction over time of a more general lateralized spatial attention disorder, with between-subject differential recovery among the different subtypes of neglect tested. However, were this the case, one would expect that the individual shifts in patient performance would be consistent with recovery; patients would not be expected to manifest new subtype symptoms over time.

Our finding of a relative increase over time in the number of patients with lateralized deficits in intention stands in contrast to prior reports that lateralized deficits of action and intention are often more transient than other subtype symptoms (Kerkhoff, 2001). For example, Mattingley and colleagues (1994) tested 13 patients with stroke and new onset of neglect and retested them after a year, and found that patients with directional hypokinesia and bradykinesia often had recovery of these symptoms, particularly when associated with anterior lesions. However, cases of emerging intentional deficits have also been reported. Kwon and Heilman (1991) reported the case of motor-intentional deficits (albeit ipsilesional) in a patient that emerged weeks after initial insult. The authors posited that the patient's worsening lateralized spatial bias reflected a release of contralesional intention-related frontal cortical areas from contralateral inhibition. The mechanisms underlying the emergence of intentional neglect in our patient cohort remain unclear. 
One interpretation of the development of discrete performance patterns over time and specific emergence of intentional neglect is that, despite early inconsistencies in performance, stable subtypes of neglect emerge over time. If neglect symptoms do become more consistent over time, it may be the case that classification of subacute or chronic neglect patients according to subtypes may have better prognostic value than classification of acute stroke patients, although this remains to be demonstrated.

To our knowledge, this is the first study to assess the stability and consistency of neglect symptoms in the weeks following right hemisphere stroke. We conclude that the performance of patients with acute neglect may have dynamic properties that have been overlooked in prior studies and that future investigations may need to take these properties into account in order to more accurately characterize patients with early neglect and to better determine the validity, neural mechanisms, and prognostic value of neglect subtypes.

\section{ACKNOWLEDGMENTS}

The authors have reported no conflicts of interest. Dr. Buxbaum's participation was funded in part by a grant from the James S. McDonnell Foundation entitled "Effectiveness of Treatments of Hemispatial Neglect." The authors thank Prin Amorapanth for creating the artwork used in Figures 1a, 1b, and 1c.

\section{REFERENCES}

Anderson, B., Mennenmeier, M., \& Chatterjee, A. (2000). Variability not ability: Another basis for performance decrements in neglect. Neuropsychologia, 38, 785-796.

Appelros, P., Nydevik, I., Karlsson, G.M., Thorwalls, A., \& Seiger, A. (2004). Recovery from unilateral neglect after right-hemisphere stroke. Disability and Rehabilitation, 26, 471-477.

Bailey, M.J., Riddoch, M.J., \& Crome, P. (2004). Test-retest stability of three tests for unilateral visual neglect in patients with stroke: Star Cancellation, Line Bisection, and the Baking Tray Task. Neuropsychological Rehabilitation, 14, 403-419.

Barrett, A.M., Crucian, G.P., Schwartz, R.L., \& Heilman, K.M. (1999). Adverse effect of dopamine agonist therapy in a patient with motor-intentional neglect. Archives of Physical Medicine and Rehabilitation, 80, 600-603.

Bisiach, E. \& Luzzatti, C. (1978). Unilateral neglect of representational space. Cortex, 14, 129-133.

Bisiach, E., Perani, D., Vallar, G., \& Berti, A. (1986). Unilateral neglect: Personal and extrapersonal. Neuropsychologia, 24, 759-767.

Bowen, A., McKenna, K., \& Tallis, R.C. (1999). Reasons for variability in the reported rate of occurrence of unilateral spatial neglect after stroke. Stroke, 30, 1196-1202.

Brass, M., Ullsperger, M., Knoesche, T.R., von Cramon, D.Y., \& Phillips, N.A. (2005). Who comes first? The role of the prefrontal and parietal cortex in cognitive control. Journal of Cognitive Neuroscience, 17, 1367-1375.

Buxbaum, L.J., Ferraro, M.K., Veramonti, T., Farne, A., Whyte, J.,
Ladavas, E., Frassinette, F., \& Coslett, H.B. (2004). Hemispatial neglect: Subtypes, neuroanatomy, and disability. Neurology, 62, 749-756.

Buxbaum, L.J. \& Permaul, P. (2001). Hand-centered attentional and motor asymmetries in unilateral neglect. Neuropsychologia, 39, 653-664.

Cocchini, G., Beschin, N., \& Jehkonen, M. (2001). The Fluff Test: A simple task to assess body representation neglect. Neuropsychological Rehabilitation, 11, 17-31.

Coslett, H.B. (1999). Spatial influences on motor and language function. Neuropsychologia, 37, 695-706.

Coslett, H.B., Bowers, D., Fitzpatrick, E., Haws, B., \& Heilman, K.M. (1990). Directional hypokinesia and hemispatial inattention in neglect. Brain, 113, 475-486.

Coslett, H.B., Bowers, D., \& Heilman, K.M. (1987). Reduction in cognitive activation after right hemisphere stroke. Neurology, 37, 957-962.

Duncan, J., Bundesen, C., Olson, A., Humphreys, G., Chavda, S., \& Shibuya, H. (1999). Systematic analysis of deficits in visual attention. Journal of Experimental Psychology: General, 128, 450-78.

Fimm, B., Willmes, K., \& Spijkers, W. (2006). The effect of low arousal on visuo-spatial attention. Neuropsychologia, 44, 1261-1268.

Guariglia, C. \& Antonucci, G. (1992). Personal and extrapersonal space: A case of neglect dissociation. Neuropsychologia, 30, 1001-1010.

Harvey, M., Kramer-McCaffery, T., Dow, L., Murphy, P.J., \& Gilchrist, I.D. (2002). Categorisation of "perceptual" and "premotor" neglect patients across different tasks: Is there strong evidence for a dichotomy? Neuropsychologia, 40, 1387-1395.

Heilman, K.M., Valenstein, E., \& Watson, R.T. (2000). Neglect and related disorders. Seminars in Neurology, 20, 463-470.

Heilman, K.N., Watson, R.T., \& Valenstein, E. (1985). Neglect and related disorders. In K.M. Heilman \& E. Valenstein (Eds.), Clinical Neuropsychology. New York: Oxford Press.

Hjaltason, H., Tegner, R., Tham, K., Levander, M., \& Ericson, K. (1996). Sustained attention and awareness of disability in chronic neglect. Neuropsychologia, 34, 1229-1233.

Karnath, H.O., Ferber, S., \& Himmelbach, M. (2001). Spatial awareness is a function of the temporal and not the posterior parietal lobe. Nature, 21, 950-953.

Katz, N., Hartman-Maeir, A., Ring, H., \& Soroker, N. (1999). Functional disability and rehabilitation outcome in right hemisphere damage patients with and without unilateral spatial neglect. Archives of Physical Medicine and Rehabilitation, 80, 379-384.

Kerkhoff, G. (2001). Spatial hemineglect in humans. Progress in Neurobiology, 63, 1-27.

Kwon, S.E. \& Heilman, K.M. (1991). Ipsilateral neglect in a patient following a unilateral frontal lesion. Neurology, 41, 2001-2004.

Levy, D., Blizzard, R.A., Halligan, P.W., \& Stone, S.P. (1995). Fluctuations in visual neglect after stroke? European Neurology, 35, 341-343.

Losier, B.J.W. \& Klein, R.M. (2001). A review of the evidence for a disengage deficit following parietal lobe damage. Neuroscience and Biobehavioral Reviews, 25, 1-13.

Malhotra, P.A., Parton, A.D., Greenwood, R., \& Husain, M. (2006). Noradrenergic modulation of space exploration in visual neglect. Annals of Neurology, 59, 186-190.

Mattingley, J.B., Bradshaw, J.L., Bradshaw, J.A., \& Nettleton, N.C. (1994). Recovery from directional hypokinesia and brady- 
kinesia in unilateral neglect. Journal of Clinical and Experimental Neuropsychology, 16, 861-876.

Na, D.L., Adair, J.C., Kang, Y., Chung, C.S., Lee, K.H., \& Heilman, K.M. (1999). Motor perseverative behavior on a line cancellation task. Neurology, 52, 1569-1576.

Paolucci, S., Antonucci, G., Grasso, M.G., \& Pizzamiglio, L. (2001). The role of unilateral spatial neglect in rehabilitation of right brain-damaged ischemic stroke patients: A matched comparison. Archives of Physical Medicine and Rehabilitation, 36, 743-749.

Pizzamiglio, L., Committeri, G., Galati, G., \& Patria, F. (2000). Psychophysical properties of line bisection and body midline perception in unilateral neglect. Cortex, 2000, 469-484.

Posner, M.I., Walker, J.A., Friedrich, F.J., \& Rafal, R.D. (1984). Effects of parietal injury on covert orienting of attention. Journal of Neuroscience, 4, 1863-1874.

Rusconi, M.L., Maravita, A., Bottini, G., \& Vallar, G. (2002). Is the intact side really intact? Perseverative responses in patients with unilateral neglect: A productive manifestation. Neuropsychologia, 40, 594-604.

Robertson, I.H. (2001). Do we need the "lateral" in unilateral neglect? Spatially nonselective deficits in unilateral neglect and their implications for rehabilitation. NeuroImage, 14, S85-S90.

Small, M. \& Ellis, S. (1994). Brief remission periods in visuospatial neglect: Evidence from long-term follow-up. European $\mathrm{Neu}$ rology, 34, 147-154.
Sterzi, R., Bottini, G., Celani, M.G., Righetti, E., Lamassa, M., Ricci, R., \& Vallar, G. (1993). Hemianopia, hemiananaesthesia, and hemiplegia after right and left hemisphere damage. A hemispheric difference. Journal of Neurology, Neurosurgery \& Psychiatry, 56, 308-310.

Stone, S.P., Patel, P., Greenwood, R.J., \& Halligan, P.W. (1992). Measuring visual neglect in acute stroke and predicting its recovery: The visual neglect recovery index. Journal of Neurology, Neurosurgery \& Psychiatry, 55, 431-436.

Sturm, W. \& Willmes, K. (2001). On the functional neuroanatomy of intrinsic and phasic alertness. NeuroImage, 14, S76-S84.

Vallar, G. (2001). Extrapersonal visual unilateral spatial neglect and its neuroanatomy. NeuroImage, 14, S52-S58.

Vallar, G. \& Perani, D. (1986). The anatomy of unilateral neglect after right-hemisphere stroke lesions. A clinical/CT scan correlation study in man. Neuropsychologia, 24, 609-622.

Welbourne, S.R. \& Lambon Ralph, M.A. (2005a). Exploring the impact of plasticity-related after brain damage in a connectionist model of single-word reading. Cognitive, Affective, \& Behavioral Neuroscience, 5, 77-92.

Welbourne, S.R. \& Lambon Ralph, M.A. (2005b). Using computational, parallel distributed processing networks to model rehabilitation in patients with acquired dyslexia: An initial investigation. Aphasiology, 19, 789-806.

Wilson, B., Cockburn, J., \& Halligan, P. (1987). Development of a behavioral test of visuospatial neglect. Archives of Physical Medicine and Rehabilitation, 68, 98-102.

Appendix 1: Individual subject performance data

\begin{tabular}{|c|c|c|c|c|c|c|c|c|c|c|c|c|}
\hline Subject & Session & $\begin{array}{l}\text { Days } \\
\text { Post- } \\
\text { stroke }\end{array}$ & $\begin{array}{l}\text { LR } \\
\% \\
\text { diff }\end{array}$ & $\begin{array}{l}\text { Distance } \\
\text { from } \\
\text { cutoff }\end{array}$ & $\begin{array}{c}\text { Int } \\
\text { Neglect? }\end{array}$ & $\begin{array}{l}\text { LT } \\
\% \\
\text { diff }\end{array}$ & $\begin{array}{l}\text { Distance } \\
\text { from } \\
\text { cutoff }\end{array}$ & $\begin{array}{c}\text { Att } \\
\text { Neglect? }\end{array}$ & $\begin{array}{l}\text { Fluff } \\
\text { Score }\end{array}$ & $\begin{array}{l}\text { Distance } \\
\text { from } \\
\text { cutoff }\end{array}$ & $\begin{array}{c}\text { Per } \\
\text { Neglect? }\end{array}$ & Subtype? \\
\hline \multirow[t]{3}{*}{1} & 1 & 34 & -31.6 & 20.5 & YES & -33.6 & 3.6 & YES & 2.0 & 1.0 & YES & All \\
\hline & 2 & 41 & -33.1 & 22.0 & YES & -28.4 & -1.6 & NO & 2.0 & 1.0 & YES & Int/Per \\
\hline & 3 & 48 & -20.4 & 9.3 & YES & -7.8 & -22.2 & NO & 6.0 & -3.0 & $\mathrm{NO}$ & Int \\
\hline \multirow[t]{3}{*}{2} & 1 & 11 & -12.7 & 1.6 & YES & -39.3 & 9.3 & YES & 1.0 & 2.0 & YES & All \\
\hline & 2 & 25 & -6.8 & -4.3 & NO & -15.6 & -14.4 & NO & 6.0 & -3.0 & NO & None \\
\hline & 3 & 32 & 1.4 & -12.5 & NO & -39.2 & 9.2 & YES & 5.0 & -2.0 & NO & Att \\
\hline \multirow[t]{3}{*}{3} & 1 & 16 & -10.8 & -0.3 & NO & -55.9 & 25.9 & YES & 4.0 & -1.0 & YES & Att/Per \\
\hline & 2 & 23 & -24.3 & 13.2 & YES & -27.6 & -2.4 & NO & 2.0 & 1.0 & YES & Int/Per \\
\hline & 3 & 37 & -1.6 & -9.5 & NO & -12.1 & -17.9 & NO & 4.0 & -1.0 & YES & Per \\
\hline \multirow[t]{3}{*}{4} & 2 & 23 & 5.0 & -16.1 & NO & -45.7 & 15.7 & YES & 4.0 & -1.0 & YES & Att/Per \\
\hline & 3 & 30 & -2.3 & -8.8 & NO & -0.9 & -29.1 & NO & 6.0 & -3.0 & NO & None \\
\hline & 3 & 37 & 9.4 & -20.5 & NO & -44.1 & 14.1 & YES & 5.0 & -2.0 & NO & Att \\
\hline \multirow[t]{3}{*}{5} & 1 & 26 & 0.8 & -11.9 & NO & -35.6 & 5.6 & YES & 4.0 & -1.0 & YES & Att/Per \\
\hline & 3 & 40 & 15.2 & -26.3 & NO & -24.7 & -5.3 & NO & 6.0 & -3.0 & NO & None \\
\hline & 3 & 166 & 12.6 & -23.7 & NO & -5.4 & -24.6 & $\mathrm{NO}$ & 6.0 & -3.0 & NO & None \\
\hline \multirow[t]{3}{*}{6} & 1 & 15 & -33.3 & 22.2 & YES & -52.3 & 22.3 & YES & 6.0 & -3.0 & NO & Att/Int \\
\hline & 2 & 22 & -26.0 & 14.9 & YES & -10.1 & -19.9 & NO & 6.0 & -3.0 & NO & Int \\
\hline & 3 & 74 & 23.3 & -34.4 & NO & -17.6 & -12.4 & NO & 6.0 & -3.0 & NO & None \\
\hline \multirow[t]{3}{*}{7} & 1 & 13 & -26.7 & 15.6 & YES & -55.6 & 25.6 & YES & 6.0 & -3.0 & NO & Att/Int \\
\hline & 2 & 20 & -12.2 & 1.1 & YES & -20.9 & -9.1 & NO & 6.0 & -3.0 & NO & Int \\
\hline & 3 & 104 & 1.0 & -12.1 & NO & -10.9 & -19.1 & NO & 6.0 & -3.0 & NO & None \\
\hline \multirow[t]{3}{*}{8} & 1 & 21 & 9.3 & -20.4 & NO & -47.9 & 17.9 & YES & 6.0 & -3.0 & NO & Att \\
\hline & 2 & 28 & 1.0 & -12.1 & NO & -50.2 & 20.2 & YES & 6.0 & -3.0 & NO & Att \\
\hline & 3 & 35 & 3.1 & -14.2 & NO & -33.0 & 3.0 & YES & 6.0 & -3.0 & NO & Att \\
\hline \multirow[t]{3}{*}{9} & 1 & 36 & -9.5 & -1.6 & NO & -31.5 & 1.5 & YES & 6.0 & -3.0 & NO & Att \\
\hline & 2 & 43 & -13.2 & 2.1 & YES & -16.3 & -13.7 & NO & 6.0 & -3.0 & NO & Int \\
\hline & 3 & 50 & -5.3 & -5.8 & NO & -8.1 & -21.9 & NO & 4.0 & -1.0 & YES & $\begin{array}{c}\text { Per } \\
\text { continued) }\end{array}$ \\
\hline
\end{tabular}


Appendix 1 (continued)

\begin{tabular}{|c|c|c|c|c|c|c|c|c|c|c|c|c|}
\hline Subject & Session & $\begin{array}{l}\text { Days } \\
\text { Post- } \\
\text { stroke }\end{array}$ & $\begin{array}{c}\text { LR } \\
\% \\
\text { diff }\end{array}$ & $\begin{array}{c}\text { Distance } \\
\text { from } \\
\text { cutoff }\end{array}$ & $\begin{array}{c}\text { Int } \\
\text { Neglect? }\end{array}$ & $\begin{array}{c}\text { LT } \\
\% \\
\text { diff }\end{array}$ & $\begin{array}{c}\text { Distance } \\
\text { from } \\
\text { cutoff }\end{array}$ & $\begin{array}{c}\text { Att } \\
\text { Neglect? }\end{array}$ & $\begin{array}{l}\text { Fluff } \\
\text { Score }\end{array}$ & $\begin{array}{c}\text { Distance } \\
\text { from } \\
\text { cutoff }\end{array}$ & $\begin{array}{c}\text { Per } \\
\text { Neglect? }\end{array}$ & Subtype? \\
\hline \multirow[t]{3}{*}{10} & 1 & 9 & 6.3 & -17.4 & $\mathrm{NO}$ & -26.5 & -3.5 & $\mathrm{NO}$ & 4.0 & -1.0 & YES & Per \\
\hline & 2 & 16 & -1.2 & -9.9 & NO & -22.5 & -7.5 & $\mathrm{NO}$ & 5.0 & -2.0 & NO & None \\
\hline & 3 & 34 & -23.7 & 12.6 & YES & -5.5 & -24.5 & NO & 6.0 & -3.0 & NO & Int \\
\hline \multirow[t]{3}{*}{11} & 1 & 37 & -20.9 & 9.8 & YES & -2.0 & -28.0 & NO & 6.0 & -3.0 & NO & Int \\
\hline & 2 & 44 & -13.0 & 1.9 & YES & 11.0 & -41.0 & NO & 6.0 & -3.0 & NO & Int \\
\hline & 3 & 51 & -0.6 & -10.5 & NO & 16.7 & -46.7 & $\mathrm{NO}$ & 6.0 & -3.0 & NO & None \\
\hline \multirow[t]{3}{*}{12} & 1 & 5 & -1.1 & -10.0 & $\mathrm{NO}$ & -7.3 & -22.7 & NO & 6.0 & -3.0 & NO & None \\
\hline & 2 & 13 & 5.9 & -17.0 & NO & -58.6 & 28.6 & YES & 5.0 & -2.0 & NO & Att \\
\hline & 3 & 287 & 5.3 & -16.4 & NO & -31.4 & 1.4 & YES & 6.0 & -3.0 & NO & Att \\
\hline \multirow[t]{3}{*}{13} & 1 & 27 & -1.5 & -9.6 & $\mathrm{NO}$ & 3.0 & -33.0 & NO & 5.0 & -2.0 & NO & None \\
\hline & 2 & 34 & -20.1 & 9.0 & YES & -33.0 & 3.0 & YES & 6.0 & -3.0 & NO & Att/Int \\
\hline & 3 & 41 & -2.2 & -8.9 & NO & -11.6 & -18.4 & NO & 6.0 & -3.0 & $\mathrm{NO}$ & None \\
\hline \multirow[t]{3}{*}{14} & 1 & 23 & -1.3 & -9.8 & $\mathrm{NO}$ & -22.2 & -7.8 & NO & 5.0 & -2.0 & NO & None \\
\hline & 2 & 30 & 0.6 & -11.7 & $\mathrm{NO}$ & -7.9 & -22.1 & NO & 4.0 & -1.0 & YES & Per \\
\hline & 3 & 37 & -5.5 & -5.6 & $\mathrm{NO}$ & 11.8 & -41.8 & NO & 6.0 & -3.0 & NO & None \\
\hline \multirow[t]{3}{*}{15} & 1 & 27 & -11.0 & -0.1 & NO & 41.1 & -71.1 & NO & 6.0 & -3.0 & NO & None \\
\hline & 1 & 19 & -20.9 & 9.8 & YES & -24.6 & -5.4 & NO & 6.0 & -3.0 & $\mathrm{NO}$ & Int \\
\hline & 2 & 34 & -17.9 & 6.8 & YES & -14.1 & -15.9 & NO & 6.0 & -3.0 & NO & Int \\
\hline \multirow[t]{3}{*}{16} & 1 & 29 & 16.3 & -27.4 & NO & -13.2 & -16.8 & NO & 5.0 & -2.0 & NO & None \\
\hline & 2 & 36 & -34.5 & 23.4 & YES & -13.2 & -16.8 & $\mathrm{NO}$ & 6.0 & -3.0 & NO & Int \\
\hline & 3 & 103 & -38.6 & 27.5 & YES & -13.1 & -16.9 & NO & 6.0 & -3.0 & NO & Int \\
\hline \multirow[t]{3}{*}{17} & 1 & 20 & -2.8 & -8.3 & NO & 9.3 & -39.3 & NO & 6.0 & -3.0 & NO & None \\
\hline & 2 & 28 & -12.5 & 1.4 & YES & -11.5 & -18.5 & NO & 6.0 & -3.0 & NO & Int \\
\hline & 3 & 34 & 1.0 & -12.1 & NO & -13.9 & -16.1 & NO & 6.0 & -3.0 & NO & None \\
\hline \multirow[t]{3}{*}{18} & 1 & 23 & -7.0 & -4.1 & NO & -5.7 & -24.3 & NO & 6.0 & -3.0 & NO & None \\
\hline & 2 & 34 & -7.3 & -3.8 & NO & -10.9 & -19.1 & NO & 6.0 & -3.0 & $\mathrm{NO}$ & None \\
\hline & 3 & 42 & -31.3 & 20.2 & YES & -5.8 & -24.2 & NO & 5.0 & -2.0 & $\mathrm{NO}$ & Int \\
\hline \multirow[t]{3}{*}{19} & 1 & 16 & 22.8 & -33.9 & NO & -11.7 & -18.3 & NO & 6.0 & -3.0 & $\mathrm{NO}$ & None \\
\hline & 2 & 23 & -6.9 & -4.2 & NO & -17.2 & -12.8 & NO & 6.0 & -3.0 & NO & None \\
\hline & 3 & 93 & -18.9 & 7.8 & YES & 28.2 & -58.2 & NO & 6.0 & -3.0 & $\mathrm{NO}$ & Int \\
\hline \multirow[t]{3}{*}{20} & 1 & 27 & 20.7 & -31.8 & NO & 3.5 & -33.5 & NO & 6.0 & -3.0 & NO & None \\
\hline & 2 & 34 & -1.7 & -9.4 & $\mathrm{NO}$ & 17.6 & -47.6 & NO & 6.0 & -3.0 & NO & None \\
\hline & 3 & 41 & 14.0 & -25.1 & $\mathrm{NO}$ & 9.1 & -39.1 & NO & 6.0 & -3.0 & NO & None \\
\hline \multirow[t]{3}{*}{21} & 1 & 23 & 10.0 & -21.1 & $\mathrm{NO}$ & 10.4 & -40.4 & NO & 6.0 & -3.0 & $\mathrm{NO}$ & None \\
\hline & 2 & 30 & 0.9 & -12.0 & NO & -16.0 & -14.0 & NO & 6.0 & -3.0 & NO & None \\
\hline & 3 & 37 & -7.3 & -3.8 & $\mathrm{NO}$ & -7.0 & -23.0 & $\mathrm{NO}$ & 6.0 & -3.0 & $\mathrm{NO}$ & None \\
\hline
\end{tabular}

"Subject" = subject number (1 to 21); "Session"= testing timepoint; "Days post-stroke" = interval from right hemisphere infarct to testing timepoint; "LR\%" = percentage difference between right and left on lateralized response test; "Int Neglect?" = whether subject was categorized as having intentional neglect at that timepoint, "LT\%" = percentage difference between right and left on lateralized target test; "Att Neglect?" = whether subject was categorized as having attentional neglect at that timepoint; "Fluff score" = subject's score on a six-point modified fluff test; "Per Neglect?" = whether subject was categorized as having personal neglect at that timepoint, "Distance from cutoff" = difference between subject performance on the LR, LT, or Fluff tests and normal/abnormal cutoff points, defined by the 20th percentile of performance of a large acute stroke cohort (Buxbaum et al., 2004), "Subtype" = Category of neglect the subject was considered to have at a given timepoint based on combined performance on LR, LT, and Fluff tests; "Att" = attentional neglect; "Int" = intentional neglect; "Per" = Personal neglect; "All" = all three subtypes; "None" = no subtypes. 